\title{
Broken discrete symmetries in a frustrated honeycomb antiferromagnet
}

\author{
H. D. Rosales, ${ }^{1}$ D. C. Cabra, ${ }^{1}$ C. A. Lamas, ${ }^{2}$ P. Pujol, ${ }^{2}$ and M. E. Zhitomirsky ${ }^{3}$ \\ ${ }^{1}$ IFLP, Departamento de Física, Universidad Nacional de La Plata, La Plata, Argentina \\ ${ }^{2}$ Laboratoire de Physique Théorique, IRSAMC, CNRS and Université de Toulouse, UPS, F-31062 Toulouse, France \\ ${ }^{3}$ Service de Physique Statistique, Magnétisme et Supraconductivité, UMR-E9001 CEA-INAC/UJF, 17 rue des Martyrs, \\ 38054 Grenoble Cedex 9, France
}

(Received 8 August 2012; revised manuscript received 29 January 2013; published 7 March 2013)

\begin{abstract}
We study the magnetic phase diagram of the $J_{1}-J_{2}$ Heisenberg antiferromagnet on a honeycomb lattice at the strongly frustrated point $J_{2} / J_{1}=1 / 2$ using large-scale Monte Carlo simulations. At low temperatures we find three different field regimes, each characterized by different broken discrete symmetries. In low magnetic fields up to $h_{c 1} / J_{1} \approx 2.9$ the $Z_{3}$ rotational lattice symmetry is spontaneously broken while a $1 / 2$-magnetization plateau is stabilized around $h_{c 2} / J_{1}=4$. The collinear plateau state and the coplanar state in higher fields break the $Z_{4}$ translational symmetry and correspond to triple- $q$ magnetic structures. The intermediate phase $h_{c 1}<h<h_{c 2}$ has an interesting symmetry structure, breaking simultaneously the $Z_{3}$ and $Z_{4}$ symmetries. At much lower temperatures the spatial broken discrete symmetries coexist with the quasi-long-range order of the transverse spin components.
\end{abstract}

DOI: 10.1103/PhysRevB.87.104402

PACS number(s): $75.10 . \mathrm{Hk}$

The search for a quantum spin liquid, an insulating magnet with a gapless ground state which breaks neither lattice nor spin symmetries, has been the focus of many studies on twodimensional frustrated quantum antiferromagnets. ${ }^{1-4}$ Such systems are assumed to be the main candidates to describe a rich variety of unconventional phases, phase transitions, and critical points with deconfined fractional excitations. ${ }^{5-7}$ Frustration plays an important role in classical systems as well. Within this context, the phenomenon of order by disorder $(\mathrm{OBD})^{8}$ is the perfect example where the interplay of frustration and fluctuations produces the emergence of unexpected order. OBD implies that certain low-temperature spin configurations are favored by higher entropy rather than by lower energy. For instance, some frustrated spin models may exhibit magnetization plateaus even at the classical level..$^{9}{ }^{10}$ In this case fluctuations are responsible for stabilizing particular collinear spin configurations that have softer excitation spectra compared to a general noncollinear spin state. Another nontrivial fluctuation effect is a finite-temperature transition in two-dimensional Heisenberg antiferromagnets related to breaking (discrete) lattice symmetries in the absence of a long-range magnetic order. ${ }^{11}$ Studying various fluctuationinduced types of magnetic order is important in order to establish robustness of a hypothetical spin-liquid state.

Here we consider the Heisenberg antiferromagnet on a honeycomb lattice, a model realized in a number of real magnetic materials. ${ }^{12-15}$ Recent interest in this model is largely motivated by the experimental realization of the spin-liquid state in $\mathrm{Bi}_{3} \mathrm{Mn}_{4} \mathrm{O}_{12}\left(\mathrm{NO}_{3}\right) .{ }^{16}$ Mulder et al. ${ }^{17}$ have studied the $J_{1}-J_{2}$ frustrated honeycomb antiferromagnet [Fig. 1(a)] in zero magnetic field. They have found that quantum fluctuations select a family of special states characterized by three inequivalent $\mathbf{Q}$ vectors for $1 / 6<J_{2} / J_{1} \leqslant 1 / 2$ and by three different ones for $1 / 2<J_{2} / J_{1}$. While at finite temperatures thermal fluctuations melt the spiral order, the discrete $Z_{3}$ lattice rotational symmetry is still broken at low temperatures.

In this paper we extend the previous theoretical work on the $J_{1}-J_{2}$ honeycomb antiferromagnet to finite magnetic fields. This is important in view of the experimentally observed field-induced transition between the spin-liquid state and a long-range ordered magnetic structure in $\mathrm{Bi}_{3} \mathrm{Mn}_{4} \mathrm{O}_{12}\left(\mathrm{NO}_{3}\right) .{ }^{16}$ As the main interlayer coupling is nonfrustrating, and the spin involved is relatively high, any phase which would be due to the bilayer and/or which would be intrinsically quantum mechanical should be present only at extremely low temperatures. Then the classical description given in this paper should be valid for a very wide range of energy scales. Specifically, we focus on the highly frustrated point at $J_{2} / J_{1}=1 / 2$. Surprisingly for such a simple model, we find a plethora of new emergent broken symmetries, which may exist in magnetic fields alongside a 1/2-magnetization plateau.

Our main results are summarized in Fig. 1(c). The magnetic phase diagram at low temperatures is divided into three regions. At low fields, and very low temperatures, the spins show a quasi-long-range order (QLRO) in the $X Y$ plane in a canted antiferromgnetic (single- $q$ ) structure which breaks the translational and orientational symmetries of the lattice. By increasing the temperature while keeping fixed the value of the magnetic field, we expect a Kosterlitz-Thouless (KT) transition to a phase in which the internal structure melts down. The study of the expected KT transitions is beyond the scope of this paper. This intermediate phase has the $Z_{3}$ spatial symmetry broken, similar to what was found in Ref. 17.

When the value of the magnetic field exceeds $h_{c_{1}} / J_{1} \simeq$ 2.9 , we find a first-order transition into an intermediate phase similar to the 2-1-1 phase observed in the pyrochlore antiferromagnet $\mathrm{ZnCr}_{2} \mathrm{O}_{4}{ }^{19}$ which transforms at $h_{c_{2}} / J_{1}=4$ into the collinear 3-1 structure via a second-order transition. Although in all high field phases the unit cell of the system have eight spins, we shall use the same terminology as in Ref. 19 to emphasize the magnetic structure for each frustrated four-spin block. The collinear 3-1 state corresponds to the magnetization plateau with $M / M_{\text {sat }}=1 / 2$. It preserves the $X Y$ rotational symmetry about the field direction but breaks in a special way the discrete $Z_{4}$ lattice translational symmetry (see the inset in Fig. 2). For this value of the magnetization (magnetic field) increasing temperature produces a phase transition into the paramagnetic phase via a continuous transition in the 


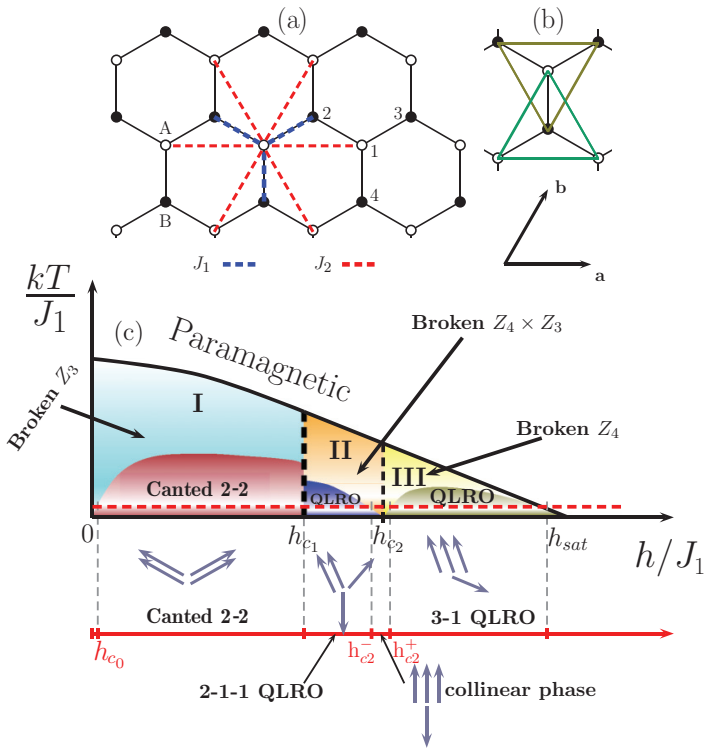

FIG. 1. (Color online) (a) Honeycomb lattice. Blue (red) line represents first (second) nearest neighbors. (b) Elementary tetrahedrons used to write the Hamiltonian in the highly frustrated point. (c) Schematic phase diagram. The dotted line represent a constant low-temperature line which can be obtained by increasing the magnetic field. By moving along this line one should observe phases I, II, and III with broken $Z_{3}, Z_{3} \times Z_{4}$, and $Z_{4}$ symmetries respectively. In phase III the lattice orientational symmetry is restored. If the temperature is low enough (as depicted in the figure) QLRO phases should be present everywhere except in the vicinity of $h=0$ and $h_{c_{2}} / J_{1}=4$ where the pseudoplateau with a collinear configuration is present. The study of the expected KT transitions is beyond the scope of this paper. Finally, increasing further the magnetic field should drive the system into the trivial paramagnetic phase characteristic of the high-temperature region.

universality class of the $Z_{4}$ clock model. It is interesting to notice that the 2-1-1 state has a supplementary $Z_{3}$ broken symmetry with respect to the 3-1 state. One would then expect again a three-states Potts transition by keeping the temperature fixed and increasing the magnetic field in order to pass from the 2-1-1 to the 3-1 state.

By increasing the applied magnetic field beyond the $1 / 2$ plateau, we enter into the high-field phase where a 3-1 state known previously for the pyrochlore antiferromagnet ${ }^{19}$ becomes stable. The classical configurations for the spins are now canted again, we have broken translational symmetry due to the long-range order in the $z$ component of the spins, and we recover QLRO order for the $X Y$ components. Note that, in contrast to the low field phase, the lattice orientational symmetry is unbroken.

To see all this in detail, let us now introduce the model. The spin Hamiltonian is given by

$$
H=J_{1} \sum_{\langle i, j\rangle_{1}} \mathbf{S}_{i} \cdot \mathbf{S}_{j}+J_{2} \sum_{\langle i, j\rangle_{2}} \mathbf{S}_{i} \cdot \mathbf{S}_{j}-h \sum_{i} S_{i}^{z},
$$

where $\langle i, j\rangle_{1}$ and $\langle i, j\rangle_{2}$ denote nearest and next nearest neighbors, respectively, $J_{1}, J_{2}>0$, and $h$ is the external magnetic field.

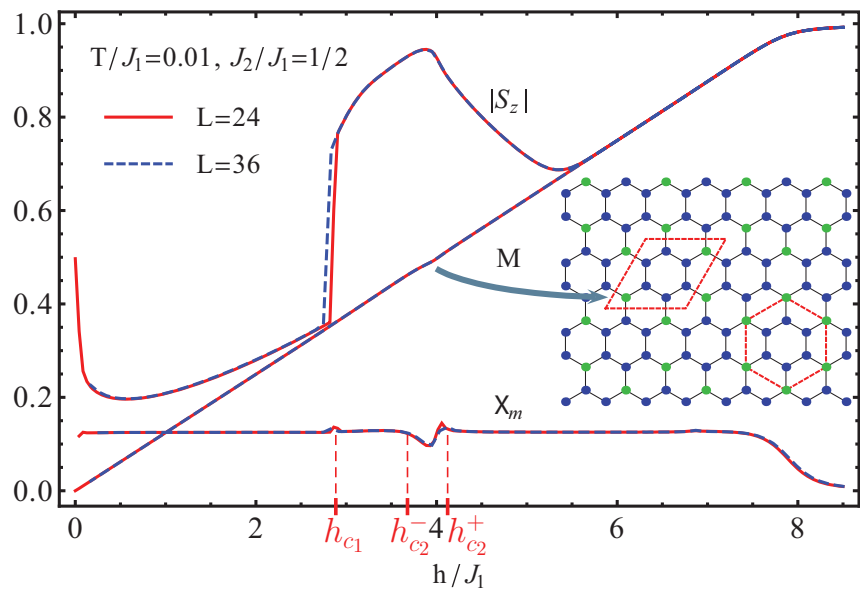

FIG. 2. (Color online) Magnetization curve, absolute value of total $S_{z}$, and susceptibility as a function of the magnetic field for $J_{2} / J_{1}=1 / 2$ for two system sizes $\left(N=2 * L^{2}\right)$. Inset: $S_{z}$ spin configuration, иuиuиudd state (collinear $3-1$ ), on the $M / M_{\text {sat }}=$ $1 / 2$ quasiplateau at $T / J_{1}=0.01$. The blue and green dots correspond to a spin fully polarized along the magnetic field and in the opposite direction, respectively.

Let us briefly discuss the magnetically ordered phases of the model (1). For small diagonal exchange $J_{2} / J_{1}<1 / 6$, classical spins form the Neel state. For $1 / 6<J_{2} / J_{1} \leqslant 1 / 2$ the classical ground states are degenerate spirals forming a closed contour around $\Gamma$ point, while for $1 / 2<J_{2} / J_{1}$, the closed contours are centered around $\mathrm{K}$ and $\mathrm{K}^{\prime}$ points. ${ }^{17}$

We now focus on the highly frustrated point $J_{2} / J_{1}=1 / 2$, where the Hamiltonian can be written, up to a constant term, as a sum over elementary tetrahedrons that we label $\vee$ and $\wedge$, respectively [see Fig. 1(b)]: ${ }^{24}$

$$
H=\frac{J_{1}}{4} \sum_{\Omega=\vee, \wedge}\left(\mathbf{S}_{\Omega}^{2}-\frac{1}{J_{1}} \mathbf{h} \cdot \mathbf{S}_{\Omega}\right)
$$

where $\mathbf{S}_{\vee}=\sum_{i \in \vee} \mathbf{S}_{i}, \mathbf{S}_{\wedge}=\sum_{i \in \wedge} \mathbf{S}_{i}$, and $\mathbf{h}=h \hat{z}$. By minimizing the energy on each $\wedge$ and $\vee$, one obtains the constraint: $\mathbf{S}_{\vee}=\mathbf{S}_{\wedge}=\mathbf{h} /\left(2 J_{1}\right)$. The classical ground state is obtained when this constraint is satisfied in every block and presents only the typical global rotation as a degeneracy. The saturation field $h_{s}$ is determined by the condition $S_{\wedge}^{z}=4$ and $S_{\vee}^{z}=4$, which gives $h_{s}=8 J_{1}$. At this value all the spins are aligned with the $z$ axis.

Monte Carlo simulations have been performed using the standard Metropolis algorithm in combination with the microcanonical over-relaxation steps; see Ref. 18 for further details. Periodic boundary conditions were implemented for $N=2 \times L^{2}$ site clusters with $L=24-72$. At every magnetic field or temperature we discarded $10^{5}$ Monte Carlo steps (MCS) for initial relaxation, and data were collected during subsequent $2 \times 10^{5} \mathrm{MCS}$. The error bars were estimated from 20 independent runs initialized by different random numbers.

Let us now discuss various physical quantities used to clarify different phases and corresponding transitions. In the first place, we calculate the magnetization, susceptibility, and 
absolute value of $S_{z}$ defined as

$$
M=\frac{1}{N} \sum_{i=1}^{N} S_{i}^{z}, \quad \chi_{m}=\frac{d M}{d h}, \quad\left|S_{z}\right|=\frac{1}{N} \sum_{i=1}^{N}\left|S_{i}^{z}\right| .
$$

In Fig. 2 we show the magnetization curve, susceptibility, and absolute value of $S_{z}$ as a function of the external field at temperature $T=0.01 J_{1}$.

The susceptibility $\chi_{m}$ shows a dip around $h=h_{c_{2}}=4 J_{1}$, which indicates the presence of a quasiplateau phase. In the same region the absolute value of $\left|S_{z}\right|$, which measures how "collinear" is the magnetic configuration, is close to one, and therefore the magnetic phase established is a "collinear phase." The situation is completely different for small fields, $\left|S_{z}\right|$ is smaller that $1 / 2$, and then the phase corresponds to a canted 2-2 antiferromagnetic (AF). Both regions are separated by a big jump in $\left|S_{z}\right|$ around $h=h_{c_{1}} \simeq 2.9 J_{1}$, indicating a first-order phase transition.

The previous results suggest that the low field phase is continuously connected with the zero field case studied in Ref. 17. Fluctuations select a commensurate wave vector corresponding to the $M$ point in the Brillouin zone (BZ). It has residual triple degeneracy. At zero magnetic field the selected structure is described by a single wave vector. To detect this single- $q$-paramagnetic phase transition we introduce a local complex order parameter $\Delta^{\alpha \beta}(\mathbf{r})^{17}$ and its averages as

$$
\begin{gathered}
\Delta^{\alpha \beta}(\mathbf{r})=\frac{1}{2} S_{A}^{\alpha}(\mathbf{r})\left[S_{B}^{\beta}(\mathbf{r})+\omega S_{B}^{\beta}(\mathbf{r}+\mathbf{b})+\omega^{2} S_{B}^{\beta}(\mathbf{r}-\mathbf{a}+\mathbf{b})\right] \\
\Delta=\left|\frac{1}{N_{c}} \sum_{\mathbf{r} \in A} \Delta^{x x}(\mathbf{r})+\Delta^{y y}(\mathbf{r})+\Delta^{z z}(\mathbf{r})\right|, \\
\Delta_{\perp}=\left|\frac{1}{N_{c}} \sum_{\mathbf{r} \in A} \Delta^{x x}(\mathbf{r})+\Delta^{y y}(\mathbf{r})\right|, \\
\Delta_{z z}=\left|\frac{1}{N_{c}} \sum_{\mathbf{r} \in A} \Delta^{z z}(\mathbf{r})\right|
\end{gathered}
$$

where the sum over $\mathbf{r}$ runs over one of the two sublattices (say, $A$ ), $\omega=e^{2 \pi i / 3}, \alpha=x, y, z$, and $\mathbf{a}, \mathbf{b}$ are the primitive translation vectors of the direct lattice (see Fig. 1). In the previous definition we have normalized to 1 the case of a perfect "two-up, two-down" collinear configuration (achievable only at $T=0$ ).

Following the standard procedure, the second-order transition between a paramagnetic phase (large- $T$ ) and a single- $q$ phase may be located by the crossing point of the corresponding Binder cumulant $U_{\Delta}$ measured for different clusters. We have used instantaneous values of (3) to measure the susceptibility $\chi_{\Delta}$ and the Binder cumulant $U_{\Delta}$ associated with this order parameter defined as

$$
\chi_{\Delta}=\frac{N_{c}}{T}\left\langle(\Delta)^{2}\right\rangle, \quad U_{\Delta}=\frac{\left\langle(\Delta)^{4}\right\rangle}{\left\langle(\Delta)^{2}\right\rangle},
$$

where $N_{c}$ is the number of unit cells. We illustrate this method in the top panel of Fig. 3 for the transition between the paramagnetic state and the single- $q$ state. The alternative approach is to study the susceptibility since the critical exponent $\eta$ is known precisely, $\eta=4 / 15 .^{20}$ In the critical
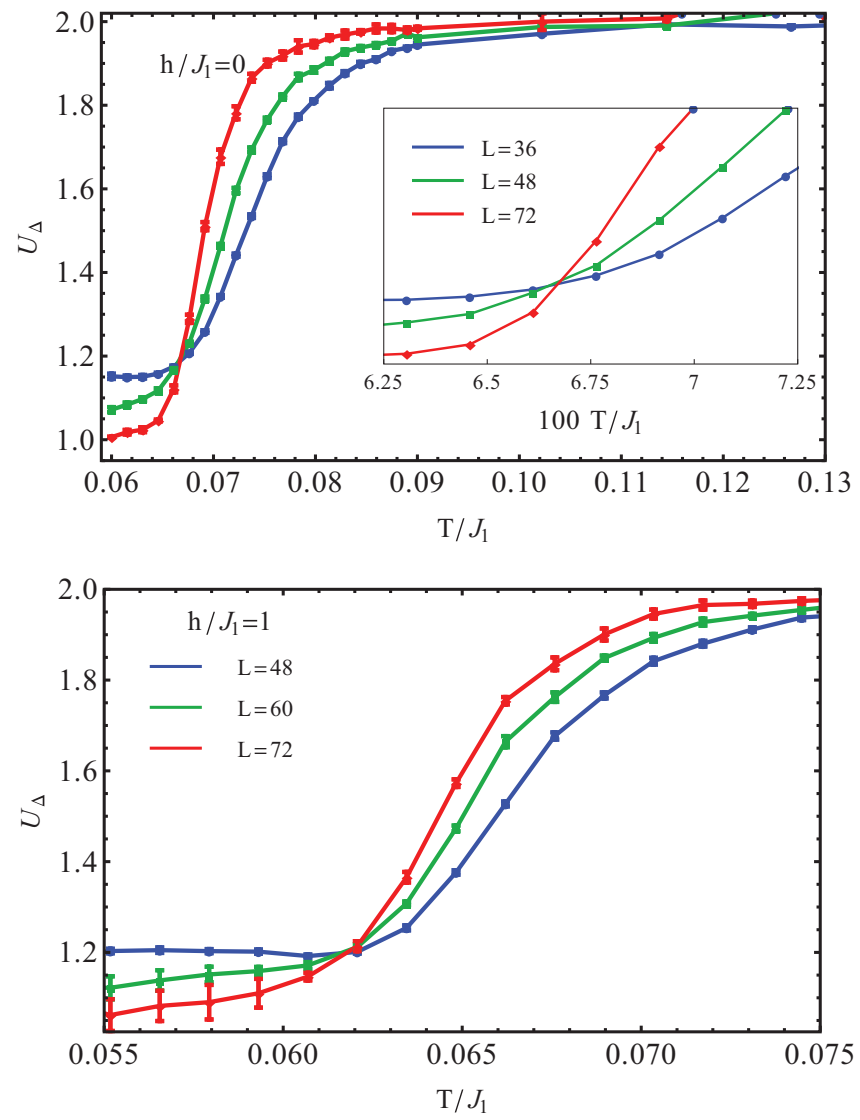

FIG. 3. (Color online) Binder cumulant associated with the order parameter $\Delta$ as a function of temperature $T$ showing the transition between the paramagnetic phase and the single- $q$ phase at $h / J_{1}=0$ (top) and $h / J_{1}=1$ (bottom), for system sizes up to $L=72$.

region the susceptibility scales as

$$
\chi_{\Delta}=L^{2-\eta} f\left(|\tau| L^{1 / \nu}\right), \quad \tau=1-T / T_{c} .
$$

Hence, the normalized susceptibility $\chi_{\Delta} / L^{2-\eta}$ becomes sizeindependent at $\tau=0$, and curves for different $L$ plotted as functions of $T$ exhibit a crossing point, similar to the behavior observed for the Binder cumulant.

In the case of nonzero field we find three different regions, as it is schematically depicted in Fig. 1:

(1) For $h<h_{c_{1}}$ we have a similar situation as for zero field; namely, at fixed magnetic field and coming from high temperature, a paramagnetic- $Z_{3}$ transition occurs. Decreasing further the temperature, one then should encounter a KT transition to a canted 2-2 AF single- $q$ QLRO in the $X Y$ spin components.

(2) For $h_{c_{1}}<h<h_{c_{2}}$ we have a finite-temperature transition related with the breaking of $Z_{4} \times Z_{3}$ spatial symmetry. Again, at low temperatures one should find a KT transition to a QLRO phase in the $X Y$ spin plane corresponding to a coplanar configuration similar to that found in Ref. 19 for the pyrochlore lattice, which we dubbed the 2-1-1 phase. Note that close to $h_{c_{1}}$, thermal fluctuations select a collinear configuration for the spins which dramatically decrease the effective spin stiffness in the $X Y$ plane when entering in the phase II. Then one expects the KT transition temperature in region II to be 


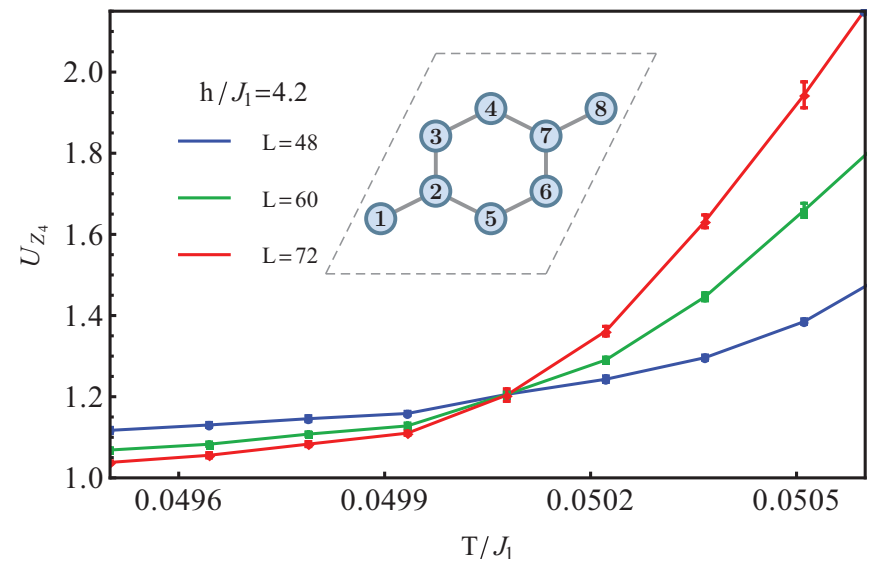

FIG. 4. (Color online) Binder cumulant corresponding to the $Z_{4}$ order parameter at $h / J_{1}=4.2$ defined in the main text. The transition between the paramagnetic phase and the 3-1 state is clearly observed.

lower than the one of region I, as schematically depicted in Fig. 1.

(3) For $h>h_{c_{2}}$, coming from the high-temperature phase, we encounter a continuous transition to a phase with broken $Z_{4}$ translational symmetry; see the inset in Fig. 2. This 3-1 phase as well as the 2-1-1 phase are characterized by mixing of three wave vectors (triple- $q$ structures). Again, at even lower temperatures, we expect a KT transition to a QLRO state where the spins adopt a planar configuration in which three spins are pointing in the same direction and the $X Y$ component of the remaining spin compensates the sum of the three first ones.

Thermal fluctuations have a strong effect in fields around $\frac{1}{2} h_{s}$, where they stabilize a collinear $3-1$, state and there is a symmetry breaking related to this selection, as we explain now. At this point the spin pattern consists of eight spins per unit cell (Fig. 2). We rewrite the coordinates of the eight spins as we show in the inset of Fig. 4 and introduce the following $Z_{4}$ order parameter:

$m^{z}=\frac{1}{N} \sum_{\square} \sum_{n=1}^{4} e^{i \frac{\pi}{2}(n-1)} S_{\square, n}^{z}+e^{i \frac{\pi}{2}(4-n)} S_{\square, n+4}^{z}$.

Using this order parameter one can construct the corresponding Binder cumulant $U_{Z_{4}}$ in the usual way. The results are shown in Fig. 4 (at $\left.h / J_{1}=4.2\right)$, measured for different cluster sizes at the transition between the paramagnetic and the $Z_{4}$ symmetry breaking collinear 3-1 state.
Results for various temperatures and magnetic field scans are summarized in the phase diagram presented in Fig. 1(c).

To summarize, we have studied the phase diagram of a strongly frustrated classical $J_{1}-J_{2}$ Heisenberg antiferromagnet on a honeycomb lattice in a magnetic field. We have found a very rich low-temperature phase diagram showing three nontrivial regions characterized by different broken lattice symmetries, as summarized in Fig. 1.

In order of increasing magnetic field at fixed (low) temperature, one first encounters a first-order transition triggered from the low field phase I, which breaks the orientational $Z_{3}$ symmetry, into the intermediate field phase II where a supplementary $Z_{4}$ symmetry, related to lattice translations, is broken. By increasing the field further, a continuous transition to phase III occurs, which provides an example of (lattice) symmetry restoration.

All these discrete broken symmetry phases should coexist with the corresponding QLRO phases, after a KT transition occurs at lower temperatures.

We have explicitly numerically checked that the transition from phase III to the paramagnetic phase is continuous, and one should then in principle expect continuously varying exponents associated with the universality class of the $Z_{4}$ Potts model. ${ }^{21}$ The richness of this phase diagram illustrates the importance of frustrated and competing interactions and the onset of fluctuation mechanisms in the selection of the low-energy configurations.

The present study may be relevant in the study of different compounds that are described by the frustrated hexagonal Heisenberg model, such as $\mathrm{Bi}_{3} \mathrm{Mn}_{4} \mathrm{O}_{12}\left(\mathrm{NO}_{3}\right),{ }^{16}$ already mentioned and the family of compounds $\mathrm{BaM}_{2}\left(\mathrm{XO}_{4}\right)_{2}$ with $\mathrm{M}$ equals; $\mathrm{Co}, \mathrm{Ni}$ and $\mathrm{X}=\mathrm{P}, \mathrm{As}$, which consist of magnetic ions $\mathrm{M}$ arranged in weakly coupled frustrated honeycomb lattices with spin $\mathrm{S}=1 / 2$ for $\mathrm{Co}$ and $\mathrm{S}=1$ for $\mathrm{Ni}^{22}$ In the case of materials with spins higher than $1 / 2$, magnetic field experiments could unravel some of the structures found in the present paper. Last but not least, the controlled setup of optical lattices for cold atoms would allow one to create arbitrary lattice structures as well as to tune the interactions. ${ }^{23}$

C.A.L. and P.P. acknowledge very fruitful discussion with N. Laflorencie. H.D.R., D.C.C., and C.A.L. are partially supported by CONICET (PIP 1691) and ANPCyT (PICT 1426). P.P. and M.E.Z. acknowledge support by the Agence Nationale de la Recherche under grants No. ANR 2010 BLANC 0406-0 and No. ANR-09-Blanc-0211, respectively.
${ }^{1}$ Fa Wang, Phys. Rev. B 82, 024419 (2010).

${ }^{2}$ Z. Y. Meng, T. C. Lang, S. Wessel, F. F. Assaad, and A. Muramatsu, Nature (London) 464, 847 (2010).

${ }^{3}$ D. C. Cabra, C. A. Lamas, and H. D. Rosales, Phys. Rev. B 83, 094506 (2011).

${ }^{4}$ D. J. J. Farnell, R. F. Bishop, P. H. Y. Li, J. Richter, and C. E. Campbell, Phys. Rev. B 84, 012403 (2011).

${ }^{5}$ P. W. Anderson, Mater. Res. Bull. 8, 153 (1973).
${ }^{6}$ P. Fazekas and P. W. Anderson, Philos. Mag. 30, 432 (1974).

${ }^{7}$ K. S. Raman, R. Moessner, and S. L. Sondhi, Phys. Rev. B 72, 064413 (2005).

${ }^{8}$ J. Villain et al., J. Phys. (Paris) 41, 1263 (1980); E. F. Shender, Zh. Eksp. Teor. Fiz. 83, 326 (1982); C. L. Henley, Phys. Rev. Lett. 62, 2056 (1989).

${ }^{9}$ M. E. Zhitomirsky, A. Honecker, and O. A. Petrenko, Phys. Rev. Lett. 85, 3269 (2000); M. E. Zhitomirsky, ibid. 88, 057204 (2002). 
${ }^{10}$ Claire Lhuillier and Gregoire Misguich, Lect. Notes Phys. 595, 161 (2002)

${ }^{11}$ P. Chandra, P. Coleman, and A. I. Larkin, Phys. Rev. Lett. 64, 88 (1990)

${ }^{12}$ H. M. Rønnow, A. R. Wildes, and S. T. Bramwell, Physica B 276-278, 676 (2000).

${ }^{13}$ M. Heinrich, H.-A. Krug von Nidda, A. Loidl, N. Rogado, and R. J. Cava, Phys. Rev. Lett. 91, 137601 (2003).

${ }^{14}$ N. Rogado, Q. Huang, J. W. Lynn, A. P. Ramirez, D. Huse, and R. J. Cava, Phys. Rev. B 65, 144443 (2002).

${ }^{15}$ E. Ressouche, M. Loire, V. Simonet, R. Ballou, A. Stunault, and A. Wildes, Phys. Rev. B 82, 100408(R) (2010).

${ }^{16}$ M. Matsuda, M. Azuma, M. Tokunaga, Y. Shimakawa, and N. Kumada, Phys. Rev. Lett. 105, 187201 (2010).

${ }^{17}$ A. Mulder, R. Ganesh, L. Capriotti, and A. Paramekanti, Phys. Rev. B 81, 214419 (2010).
${ }^{18}$ M. E. Zhitomirsky, Phys. Rev. B 78, 094423 (2008).

${ }^{19}$ K. Penc, N. Shannon, H. Shiba, and Phys. Rev. Lett. 93, 197203 (2004); A. Miyata et al., J. Phys. Soc. Jpn. 80, 074709 (2011).

${ }^{20}$ F. Y. Wu, Rev. Mod. Phys. 54, 235 (1982); P. M. Chaikin and T. C. Lubensky, Principles of Condensed Matter Physics (Cambridge University Press, Cambridge, 1995).

${ }^{21}$ J. V. José, L. P. Kadanoff, S. Kirkpatrick, and D. R. Nelson, Phys. Rev. B 16, 1217 (1977).

${ }^{22}$ L. J. De Jongh, editor, Magnetic Properties of Layered Transition Metal Compounds (Kluwer, Dordrecht, 1990).

${ }^{23}$ Z.-X. Chen, H. Ma, M.-H. Chen, X.-F. Zhou, L. He, G.-C. Guo, X. Zhou, Y. Chen, and Z.-W. Zhou, Phys. Rev. A 85, 013632 (2012).

${ }^{24}$ We use the name tetrahedron to denote four-site (planar) blocks with each site interacting equally with three others. 